\title{
Zur Quantentheorie nichtrenormierbarer Wellengleichungen
}

\author{
Von W. Heisenberg \\ Aus dem Max-Planck-Institut für Physik, Göttingen \\ Otto Hahn zum 75. Geburtstag gewidmet \\ (Z. Naturforschg. 9a, 292-303 [1954]; eingegangen am 4. Februar 1954)
}

\begin{abstract}
Das in einer früheren Arbeit ${ }^{1}$ vorgeschlagene Verfahren zur Quantisierung nichtlinearer Wellengleichungen wird an einem speziellen Beispiel genauer durchgeführt. Nach allgemeinen Vorbemerkungen 1. über den Hilbert-Raum des Systems werden in 2. die ,,Fortpflanzungsfunktionen" der nichtlinearen Wellengleichung studiert. Der Zusammenhang dieser Funktionen mit den Vertauschungsfunktionen wird in 3. besprochen und die Beziehungen zu dem Regularisierungsverfahren von Pauli und Villars erörtert. In 4. wird die Masse des leichtesten Spinorteilchens näherungsweise abgeleitet und die Eigenwertgleichung für die Bose-Teilchen angegeben. In 5. werden einige Erhaltungssätze besprochen und in 6. die Konvergenz des Näherungsverfahrens erörtert und qualitative Aussagen über die Wechselwirkung der Elementarteilchen abgeleitet.
\end{abstract}

$\mathrm{D}$ a es bisher nicht gelungen ist, nichtrenormierbaren Wellengleichungen im Zusammenhang mit den üblichen Quantisierungsvorschriften einen Sinn zu geben, hat der Verfasser in einer früheren Arbeit $^{1}$ versucht, durch eine Abänderung dieser Vorschriften $\mathrm{zu}$ einer in sich widerspruchsfreien mathematischen Behandlung solcher Gleichungen zu kommen. Im folgenden soll die spezielle Wellengleichung

$$
\gamma_{\mu} \frac{\partial \psi}{\partial x_{\mu}}+l^{2} \psi\left(\psi^{+} \psi\right)=0
$$

nach den genannten Methoden untersucht, die tiefsten Eigenwerte näherungsweise bestimmt und einige physikalische Konsequenzen abgeleitet werden.

Vor der mathematischen Durchführung sollen jedoch die physikalischen Gründe, die eine Abänderung der üblichen Quantisierungsvorschriften als möglich und notwendig erscheinen lassen, ausführlicher als in der ersten genannten Arbeit dargestellt werden.

\section{Die Eigenschaften des zu (1) gehörigen Hilbert-Raums}

Einer der wesentlichsten Unterschiede zwischen der klassischen Physik und der Quantentheorie äußert sich darin, daß bei der Bestimmung eines in der Natur realisierten Quantenzustandes auch die Gesamtheit aller möglichen anderen nicht realisierten Zustände eine Rolle spielt. So wird etwa schon

${ }^{1}$ W. Heisenberg, Nachr. Akad. Wiss. Göttingen, Jahrg. 1953, Nr. 8. der tiefste Zustand des Wasserstoffatoms davon abhängen, ob der dem Atom zur Verfügung stehende Raum endlich oder unendlich ist, und für die Berechnung der Streuung von Licht an einem Atom ist die Kenntnis der ,,virtuellen Zwischenzustände“" wichtig, die doch vom Atom während des ganzen Streuprozesses niemals eingenommen werden.

Aus diesem Grunde spielen in der Quantentheorie der Wellenfelder nicht nur die Felder eine Rolle, die sich als Lösung einer Wellengleichung zum mindesten in weiten Bereichen analytisch verhalten, sondern es muß auch die ungeheure Mächtigkeit der nichtanalytischen Funktionen in irgendeiner Weise bei den ,virtuellen Zwischenzuständen" mitberücksichtigt werden. Man denke etwa an Funktionen, die den Wert 1 haben, wenn einer der Koordinatenwerte eine rationale Zahl ist, und die sonst überall verschwinden. Solche Funktionen brauchen gewöhnlich in der Physik nicht erörtert zu werden, da sie keine wirklichen physikalischen Vorgänge darstellen, aber als ,,virtuelle Zwischenzustände“ können sie bei konsequenter Anwendung der Quantentheorie gar nicht ausgeschlossen werden. Die bekannten Divergenzschwierigkeiten in der Quantentheorie der Wellenfelder dürften ihre Wurzel an dieser Stelle haben.

Die Quantentheorie in ihrer gewöhnlichen Form geht von der Annahme aus, daß für ein gegebenes Problem jeweils ein Hilbert-Raum konstruiert werden kann, in dem die Zustände des Systems durch Vektoren (oder genauer: ,Strahlen“) charakterisiert werden. Zum Beispiel kann man diesen Raum durch ein System von Eigenvektoren aufspannen, 
die Energie, Impuls und Drehimpuls auf Diagonalform bringen. Tut man dies in der Quantentheorie der Wellenfelder, z. B. für Gl. (1), so liegt es im Hinblick auf die genannte Schwierigkeit nahe, den Hilbert-Raum in zwei Teile zu teilen. Der eine Teil I soll alle Zustände enthalten, bei denen die Gesamtmasse des Systems kleiner als eine sehr große, aber endliche Grenzmasse $M_{\mathrm{g}}$ ist, der andere Teil II alle anderen. Beschränkt man sich auf den HilbertRaum I, so kann man durch die Kombination von Zuständen dieses Raumes nur Wellenfunktionen darstellen, die in einer näher zu bezeichnenden Weise ,glatt" sind. ,Glatt" bedeutet hier ungefähr: sie können keine Fourier-Komponenten enthalten, deren Wellenlänge kleiner als eine durch $M_{\mathrm{g}}$ gegebene Grenze ist. Dieser Sachverhalt wird etwas kompliziert durch den Umstand, daß es sich um eine Fourier-Analyse in vier Dimensionen handelt, daß die relativistische Invarianz zu beachten und daß schließlich die Rolle der Wechselwirkungsenergie in dieser Betrachtung schwer zu überblicken ist. Es dürfte aber nicht nötig sein, auf die genaue Definition des Wortes ,glatt" an dieser Stelle einzugehen.

Jedenfalls kann man innerhalb des Raumes I die ungeheure Mächtigkeit der nichtanalytischen Funktionen nicht darstellen, wie groß auch immer $M_{\mathrm{g}}$ gewählt werden mag. Erst durch die Hinzunahme des Raumes II umfaßt man ,grundsätzlich“ (aber wohl kaum wirklich) alle diese Möglichkeiten.

Wenn man den Anschluß an die bisherige Quantentheorie behalten will, wird es jedenfalls notwendig sein, innerhalb des Raumes I die üblichen Regeln anzuwenden, insbesondere z. B. die Gleichung

$$
\mathfrak{\Im}_{\mu} \psi(x)-\psi(x) \mathfrak{\Im}_{\mu}=-i \frac{\partial \psi(x)}{\partial x_{\mu}}
$$

( $\mathfrak{\Im}_{\mu}$ Vierervektor von Energie und Impuls) zu fordern.

Anders ist es jedoch im Raum II, von dem alle Divergenzschwierigkeiten herrühren. Die Zustände dieses Raumes können, wenn $M_{\mathrm{g}}$ groß genug gewählt wird, physikalisch nie realisiert werden. Es mag sich also herausstellen - und die bisherigen Divergenzschwierigkeiten machen das sehr wahrscheinlich - , daß ein Hilbert-Raum II im eigentlichen Sinne für die Gl. (1) nicht konstruiert werden kann. Der Versuch in der genannten früheren Arbeit ${ }^{1}$ läuft nun darauf hinaus, den Hilbert-Raum II aufzugeben und durch einen symbolischen HilbertRaum zu ersetzen, der nur durch einige allgemeine
Eigenschaften, die für die Berechnung von Zuständen im Raum I wichtig sind, charakterisiert wird. Diese Eigenschaften wären, wie sich später herausstellt, mit der Annahme eines echten HilbertRaums II gar nicht verträglich, so daß auch z. B. die Gl. (2) in diesem symbolischen Raum nicht mehr angewendet werden kann. Die Quantentheorie im üblichen Sinne soll also im Raum II aufgegeben werden, und man kann wohl nicht sagen, daß die vorliegende experimentelle Erfahrung einer solchen Annahme unmittelbar widerspräche. Erst nach einer ausführlichen Untersuchung ihrer Konsequenzen wird man entscheiden können, ob sich die Erfahrung in dieser Weise darstellen läßt.

Man kann die Grundannahmen der genannten Arbeit auch durch den Satz ausdrücken, daß die Operatoren $\psi(x)$ gar nicht in einem echten vierdimensionalen Kontinuum (mit den Koordinaten $x$ ) definiert werden, sondern daß der Raum dieser Operator-Funktionen vorher durch eine Glättungsannahme entscheidend eingeschränkt werden soll.

\section{Die Fortpflanzungsfunktionen zur Wellengleichung (1)}

Vor der quantentheoretischen Behandlung des Problems wird es zweckmäßig sein, Lösungen der klassischen Wellengleichung (1) zu studieren, die in einem Punkt singulär sind, für alle Punkte raumartigen Abstandes von diesem Punkt verschwinden und die deshalb eng verwandt sind mit den Schwingerschen Funktionen $S$ oder $\bar{S}$ oder mit den vom Verfasser im Zusammenhang mit der Mesonenerzeugung ${ }^{2}$ studierten Lösungen.

Zu diesem Zweck sei ein konstanter Spinor $a$ eingeführt und für $\psi(x)$ versuchsweise angesetzt:

$$
\begin{gathered}
\psi(x)=\left(x_{\nu} \gamma_{\nu} \chi(s)+\varphi(s)\right) a, \\
s=-x_{\nu}^{2} .
\end{gathered}
$$

Man findet dann

$$
\begin{gathered}
\psi^{+} \psi=\psi^{*} \beta \psi=a_{\nu}^{*}\left(x_{\nu}^{*} \gamma_{\nu} \chi^{*}+\varphi^{*}\right) \beta\left(x_{\nu} \gamma_{\nu} \chi+\varphi\right) a \\
a^{*} \beta\left(-x_{\nu} \gamma_{\nu} \chi^{*}+\varphi^{*}\right)\left(x_{\nu} \gamma_{\nu} \chi+\varphi\right) a
\end{gathered}
$$

und unter der Voraussetzung, daß $\chi$ und $\varphi$ reell sein sollen,

$$
\psi^{+} \psi=a^{*} \beta a\left(s \chi^{2}+\varphi^{2}\right) .
$$

Aus (1) erhält man jetzt für die beiden reellen Funktionen $\chi$ und $\varphi$ die beiden simultanen Differentialgleichungen:

\footnotetext{
${ }^{2}$ W. Heisenberg, Z. Phys. 133, 65 [1952].
} 


$$
\begin{aligned}
4 \chi+2 s \chi^{\prime}+A \varphi\left(s \chi^{2}+\varphi^{2}\right) & =0, \\
-2 \varphi^{\prime}+A \chi\left(s \chi^{2}+\varphi^{2}\right) & =0,
\end{aligned}
$$

wobei

$$
A=l^{2}\left(a^{*} \beta a\right)
$$

eine Konstante ist, die sowohl positive wie negative Werte annehmen kann. Im Grenzfall sehr kleiner Werte von $a$ und $A$ und nicht zu kleiner Werte von $s$ erhält man die Lösung $\chi \sim$ const $/ s^{2}, \varphi \sim 0$, die wieder zu den Schwingerschen Funktionen $S$ zurückführt. Diese Ansätze können aber in der unmittelbaren Umgebung der Stelle $s=0$ keine Annäherung an die wirkliche Lösung mehr darstellen.

Durch die Substitution

$\chi(s)=s^{-3 / 4} \frac{f(z)}{V|A|} ; \quad \varphi(s)=s^{-1 / 4} \frac{V|A|}{A} g(z) ; \quad z=\lg s$

gehen die Gl. (7) über in:

$$
\begin{gathered}
\frac{5}{2} f+2 f^{\prime}+g\left(f^{2}+g^{2}\right)=0, \\
\frac{1}{2} g-2 g^{\prime}+f\left(f^{2}+g^{2}\right)=0, \quad \text { oder } \\
\mathrm{d} z=-\frac{2 \mathrm{~d} f}{\frac{5}{2} f+g\left(f^{2}+g^{2}\right)}=\frac{2 \mathrm{~d} g}{\frac{1}{2} g+f\left(f^{2}+g^{2}\right)} .
\end{gathered}
$$

Eine spezielle Lösung lautet:

$$
f= \pm \sqrt{\frac{\sqrt{5}}{12}} ; \quad g=\mp \sqrt{\frac{5 \sqrt{5}}{12}} .
$$

In der unmittelbaren Umgebung dieser Punkte kann man setzen

$$
f= \pm \sqrt{\frac{\sqrt{5}}{12}}+u ; \quad g=\mp \sqrt{\frac{5 \sqrt{5}}{12}}+v
$$

und $u$ und $v$ als sehr klein annehmen. Man erhält dann in erster Näherung

$$
\begin{aligned}
2 u^{\prime}+\frac{5}{3} u+\frac{4}{3} & \sqrt{5} \cdot v=0, \\
& -2 v^{\prime}-\frac{1}{3} v+\frac{2}{3} u \sqrt{5}=0
\end{aligned}
$$

mit den Lösungen $(C$ ist eine Integrationskonstante):

$$
\begin{aligned}
& u=\operatorname{Re}\left[C\left(-\frac{2}{3} \pm 2 i\right) e^{-\frac{z}{2} \pm i z}\right], \\
& v=\operatorname{Re}\left[C \frac{2}{3} \sqrt{5} e^{-\frac{z}{2} \pm i z}\right] .
\end{aligned}
$$

Der Exponent zeigt, daß die Lösungskurven in der $f, g$-Ebene in der Nähe der Punkte (12) Spiralen sind, die sich um diese Punkte winden, wobei die Punkte im limes $z \rightarrow \infty$ oder $s \rightarrow \infty$ erreicht werden.

Im Zusammenhang mit der quantentheoretischen Behandlung sind aber andere Lösungen wichtiger, die sich für $s \rightarrow \infty$ den Schwingerschen Funktionen $\chi=$ const $/ s^{2}$ annähern. Wir entnehmen aus (15), daß diese letzteren Lösungen sich nicht etwa für $s \rightarrow 0$ den Punkten (12) annähern können.

Zeichnet man in der $f-g$-Ebene die Kurven $f^{\prime}=0$ und $g^{\prime}=0$ nach Gl. (10) ein (vgl. Abb. 1), so erkennt man, daß diese Linien die Ebene in verschiedene Teilgebiete zerlegen, in denen $\mathrm{d} f / \mathrm{d} g$ positiv $(+$, schraffiert) oder negativ (-) ist. Man kann damit qualitativ den möglichen Verlauf von Lösungskurven auffinden (z. B. die ausgezogenen Spiralen in Abb. 1). Insbesondere zeigt sich, daß alle

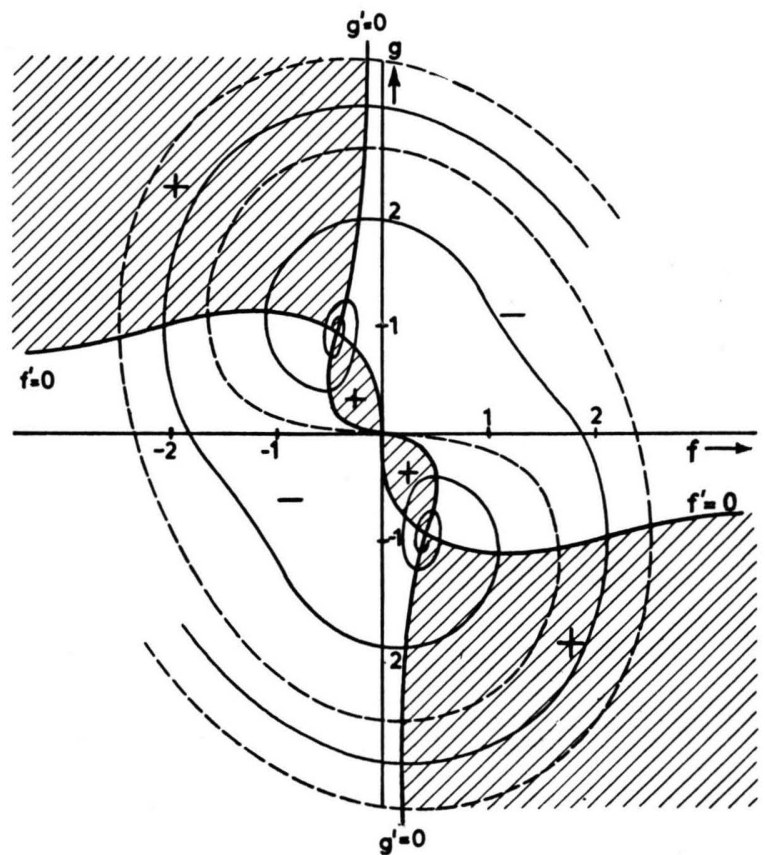

Abb. 1. Lösungskurven für Gl. (10) in $\operatorname{der} f-g$-Ebene.

Lösungskurven, z. B. auch die (in der Abb. gestrichelt angedeutete) Kurve, die für $s \rightarrow \infty$ der Schwingerschen Funktion $S_{1}$ entspricht:

$f \approx b e^{-5 z / 4} ; \quad g \approx-\frac{l^{3}}{8} e^{-15 z / 4}$ für $z \rightarrow \infty, s \rightarrow \infty$

für kleine Werte von $s$ (d.h. $z \rightarrow-\infty)$ in eine ins Unendliche laufende Spirale übergeht, die den Ursprung und die Punkte (12) umkreist. Eine Ausnahme von dieser Regel bildet nur die (in Abb. 1 nicht eingezeichnete) bei $s=0$ reguläre Lösung, bei der $f$ und $g$ für $s \rightarrow 0$ verschwinden; für diese gilt:

$$
f \approx \frac{b^{3}}{4} e^{3 z / 4} ; g \approx-b e^{z / 4} \text { für } z \rightarrow-\infty,
$$

während $f$ und $g$ sich den Punkten (12) für große $z$ annähern. $\varphi(s)$ und $\chi(s)$ gehen hier für $s \rightarrow 0$ in von Null verschiedene Grenzwerte über und gehen stetig 
über den Punkt $s=0$ hinweg, verschwinden also auch nicht überall im Gebiet negativer $s$-Werte. Alle übrigen Lösungen laufen aber für $z \rightarrow-\infty$ in die genannte Spirale aus. Wir untersuchen noch die Eigenschaften dieser Spirale.

Hierfür können $f$ und $g$ in Gl. (10) als groß angenommen und daher in gröbster Näherung die Ausdrücke $\frac{5}{2} f$ und $\frac{1}{2} g$ auf der linken Seite von (10) gegen die Ausdrücke dritten Grades vernachlässigt werden. Tut man dies, so erhält man als Näherungslösung ( $c=$ const und $c \gg 1)$ :

$f=c \cos \frac{c^{2}}{2}\left(z-z_{0}\right) ; \quad g=c \sin \frac{c^{2}}{2}\left(z-z_{0}\right)$,

also keine Spirale, sondern eine reine Kreisbewegung um den Ursprung. Man untersucht nun die Abweichungen von dieser Näherungslösung, indem man ähnlich wie in (13) ansetzt:

$f=c \cos \frac{c^{2}}{2}\left(z-z_{0}\right)+u ; \quad g=c \sin \frac{c^{2}}{2}\left(z-z_{0}\right)+v ;$

$u$ und $v$ sind dann klein von der Ordnung $1 / c$, man kann also in (10) höhere Potenzen von $u$ und $v$ vernachlässigen. In dieser Näherung erhält man mit der Abkürzung

$$
y=\frac{c^{2}}{2}\left(z-z_{0}\right)
$$

aus (10) nach der Methode der Variation der Konstanten die Lösungen:

$$
\begin{aligned}
c \cdot u(y)= & -\left(y^{2}+\frac{3}{2} y \sin 2 y\right) \sin y \\
& -\left(y+\frac{3}{4} \quad \sin 2 y\right)(\cos y-2 y \sin y), \\
c \cdot v(y) & =\left(y^{2}+\frac{3}{2} y \sin 2 y\right) \cos y \\
& -\left(y+\frac{3}{4} \sin 2 y\right)(\sin y+2 y \cos y),
\end{aligned}
$$

und insbesondere die Werte

$$
u( \pm \pi)= \pm \frac{\pi}{c} ; \quad v( \pm \pi)=\frac{\pi^{2}}{c} .
$$

Hieraus erkennt man, daß es sich um eine Spiralbewegung handelt und daß die Spirale mit abnehmendem $y$ nach außen läuft. Bei einem Umlauf ändert sich $z$ um $4 \pi / c^{2}$, und der Radius der Spirale nimmt dabei nach (21) um $2 \pi / c$ zu bzw. ab. Man kann also für die Zunahme des Radius in der Spirale näherungsweise setzen:

$$
\begin{aligned}
\frac{\mathrm{d} c}{\mathrm{~d} z} & =-\frac{2 \pi}{c} / \frac{4 \pi}{c^{2}}=-\frac{c}{2} ; \\
c & =d e^{-z / 2}=\frac{d}{\sqrt{s}},(d=\text { const }) .
\end{aligned}
$$

Die Funktionen $f$ und $g$ verhalten sich also für alle Lösungen (mit Ausnahme der bei $s=0$ regulären) in der Grenze $s \rightarrow 0$ wie

$$
\begin{aligned}
& f=d e^{-z / 2} \cos \frac{d^{2} e^{-z}}{2}\left(z-z_{0}\right) ; \\
& g=d e^{-z / 2} \sin \frac{d^{2} e^{-z}}{2}\left(z-z_{0}\right)
\end{aligned}
$$

und schließlich

$$
\begin{aligned}
& \underset{s \rightarrow 0}{\chi(s)}=\frac{d}{V|A|} s^{-5 / 4} \cos \left(\frac{d^{2}}{2 s} \lg \frac{s}{s_{0}}\right) \\
& \underset{s \rightarrow 0}{\varphi(s)}=\frac{d V|A|}{A} s^{-3 / 4} \sin \left(\frac{d^{2}}{2 s} \lg \frac{s}{s_{0}}\right) .
\end{aligned}
$$

Die Funktionen oszillieren in der Umgebung von $s=0$ beliebig rasch, der Punkt $s=0$ ist ein wesentlich singulärer Punkt. Für $s<0$ verschwinden $\chi$ und $\varphi$. Das Integral über diese Funktionen bis zur Grenze $s=0$ herunter konvergiert, die unmittelbare Umgebung von $s=0$ trägt wegen der schnellen Oszillationen nur noch sehr wenig bei. An diesem Ergebnis würde sich auch nichts ändern, wenn in Gl. (1) noch ein Massenglied $\varkappa \psi$ zugefügt würde, da dies in der Grenze $s \rightarrow 0$ keine Rolle spielt.

3. Das Verhalten der Vertauschungsfunktionen in der unmittelbaren Umgebung des singulären Punktes

Zum Studium derVertauschungsfunktionen untersuchen wir unter Korrektur eines ähnlichen Ansatzes in der früheren Arbeit ${ }^{1}$ den Operator

$$
\begin{array}{r}
\chi_{\alpha}\left(x, x^{\prime}\right)=e^{-i\left[a_{\nu} \psi_{\nu}^{+}\left(x^{\prime}\right)+\psi_{\nu}\left(x^{\prime}\right) a_{\nu}^{+}\right]} \\
\psi_{\alpha}(x) e^{+i\left[a_{\nu} \psi_{\nu}^{+}\left(x^{\prime}\right)+\psi_{\nu}\left(x^{\prime}\right) a_{\nu}^{+}\right]},
\end{array}
$$

der ebenso wie $\psi_{\alpha}(x)$ der Operator-Wellengleichung (1) genügt*. $a_{v}$ bezeichnet wieder einen konstanten Spinor, der jetzt mit allen Wellenfunktionen antikommutieren soll. Man kann ihn etwa darstellen als Produkt eines willkürlichen $c$-Zahlspinors mit einer Vorzeichenfunktion $(-1)^{\Sigma N}$, wobei $\Sigma N$ im Exponenten die gesamte „Ladung“ (vgl. Abschn. 5) bedeutet. Die Reihenentwicklung von $\chi_{\alpha}\left(x, x^{\prime}\right)$ nach den $a_{v}$ beginnt mit den Gliedern

$$
\begin{aligned}
& \chi_{\alpha}\left(x, x^{\prime}\right)=\psi(x)-i a_{\nu}\left[\psi_{\alpha}(x) \psi_{\nu}^{+}\left(x^{\prime}\right)+\psi_{\nu}^{+}\left(x^{\prime}\right) \psi_{\alpha}(x)\right] \\
& \quad+i a_{\nu}^{+}\left[\psi_{\alpha}(x) \psi_{\nu}\left(x^{\prime}\right)+\psi_{\nu}\left(x^{\prime}\right) \psi_{\alpha}(x)\right]+\ldots, \quad(26)
\end{aligned}
$$

* In der früheren Arbeit war irrtümlich für $\chi\left(x x^{\prime}\right)$ ein etwas anderer Ansatz gewählt worden, der aber, da dort die Exponentialfunktionen nicht unitär sind, die Gl. (1) nicht befriedigt. Die weiteren Schlüsse der früheren Arbeit bleiben jedoch bei der verbesserten Definition von $\chi\left(x x^{\prime}\right)$ davon unberührt. 
wobei die höheren Glieder wieder nur die Vertauschungen der Wellenfunktion mit dem Vertauschungsausdruck enthalten. Hieraus und aus der Tatsache, daß der Vertauschungsausdruck für raumartige Abstände verschwinden soll, geht schon die enge Verwandtschaft der Funktion $\chi_{\alpha}\left(x, x^{\prime}\right)$ mit den in Abs. 2 studierten Lösungen hervor.

Es liegt daher nahe, den Ansatz

$$
\chi_{\alpha}\left(x, x^{\prime}\right)=\chi_{\alpha}^{0}\left(x, x^{\prime}\right)+c_{\alpha}\left(x-x^{\prime}\right)
$$

$\mathrm{zu}$ versuchen; $\mathrm{c}_{\alpha}\left(x-x^{\prime}\right)$ soll eine Funktion von $x-x^{\prime}$ sein, in der die Operatoren der Feldgrößen nicht mehr vorkommen, die also bis auf die als Faktor auftretende Vorzeichenfunktion die Rolle einer $c$-Zahl spielt. In der Nähe des kritischen Punktes $s=0$ soll sie das singuläre Verhalten von $\chi_{\alpha}\left(x, x^{\prime}\right)$ richtig darstellen, während $\chi_{\alpha}^{0}\left(x, x^{\prime}\right)$ ein Operator ist, der in der Nähe dieses Punktes ,,glatter" verlaufen soll als $c_{\alpha}\left(x-x^{\prime}\right)$. Insbesondere könnte man etwa $c_{\alpha}\left(x-x^{\prime}\right)$ dem Vakuumerwartungswert von $\chi_{\alpha}\left(x, x^{\prime}\right)$ gleichsetzen. Über die Berechtigung und die physikalische Bedeutung des für die ganze Theorie entscheidenden Ansatzes (27) wird weiter unten noch zu sprechen sein.

Setzt man, etwa unter der Voraussetzung, daß der Vakuumserwartungswert von $\chi_{\alpha}^{0}\left(x, x^{\prime}\right)$ verschwindet, Gl. (27) in Gl. (1) ein, so wird man annehmen können, daß auch der Vakuumerwartungswert des Ausdrucks vom dritten Grade in $\chi_{\alpha}^{0}\left(x, x^{\prime}\right)$ verschwindet oder jedenfalls bei $s=0$ glatter ist als der entsprechende Ausdruck in $c\left(x-x^{\prime}\right)$, und man erhält für $c\left(x-x^{\prime}\right)$ die Gleichung

$$
\gamma_{\mu} \frac{\partial c}{\partial x_{\mu}}+l^{2} c\left(c^{+} c\right)+c \varkappa(s)=0 .
$$

Der Ausdruck $\varkappa(s)$ ist durch denVakuumerwartungswert eines in $\chi_{\alpha}^{0}\left(x, x^{\prime}\right)$ quadratischen Gliedes gegeben, der aber im folgenden durch eine konstante Masse $*$ ersetzt werden soll, da das letzte Glied in (28) auf das Verhalten von $c$ am singulären Punkt doch keinen Einfluß hat. Einstweilen soll uns vor allem das Verhalten von $c$ in der Nähe von $s=0$ interessieren.

Offenbar entspricht $c$ genau den in Abs. 2 studierten Lösungen. Für große $s$ geht es in die

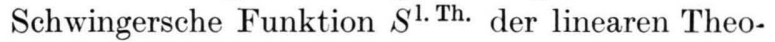
rie für die Masse $\varkappa$ über

$c_{\alpha}\left(x-x^{\prime}\right) \rightarrow-a_{\nu} S_{\alpha v}^{\text {l. Th. }}\left(x-x^{\prime}\right)$ für große $s$,

der Faktor von $a^{+}$verschwindet an dieser Grenze; für $s \rightarrow 0$ verhält es sich wie in Gl. (24) angegeben.
Der Vakuumerwartungswert derVertauschungsfunktion kann nach (26) aus

$$
S_{\alpha \nu}\left(x-x^{\prime}\right)=-\lim _{a_{\nu} \rightarrow 0} \frac{\partial c_{\alpha}\left(x-x^{\prime}\right)}{\partial a_{\nu}}
$$

berechnet werden, während der Vakuumerwartungswert von $\psi(x) \psi\left(x^{\prime}\right)$ wegen des Erhaltungssatzes der Ladung allgemein verschwindet. Dabei wird man, wenn man $c_{\alpha}\left(x-x^{\prime}\right)$ aus Gl. (28) einsetzt, das Verhalten von $S$ in der Nähe des singulären Punktes richtig treffen, weiter außen sind wegen der Vereinfachung $x=$ const Abweichungen zu erwarten; es wird aber im Näherungsverfahren zur Bestimmung der Eigenwerte auf das Verhalten weiter außen nur wenig ankommen.

Wenn man den Grenzübergang $a_{v} \rightarrow 0$ in Gl. (30) durchführt, so wird sich, wie schon in der früheren Arbeit ${ }^{1}$ erläutert, das Oszillationsgebiet der Funktion immer näher an den Punkt $s=0$ heranschieben, so daß sich in der Grenze

$$
\begin{array}{ll}
S_{\alpha \nu}\left(x-x^{\prime}\right) \approx S_{\alpha \nu}^{\mathrm{l} . T h} \cdot\left(x-x^{\prime}\right) & \text { für } s>0, \\
S_{\alpha \nu}\left(x-x^{\prime}\right)=0 \quad \text { für } s<0, & \text { unbestimmt }
\end{array}
$$

ergibt. Der Unterschied zwischen der linearen Theorie und der hier versuchten besteht also nur noch darin, daß die $\delta$ - bzw. $\delta^{\prime}$-Funktion an der Stelle $s=0$ weggefallen ist; denn die unmittelbare Umgebung von $s=0$ trägt in den Lösungen (24) nichts zum Integral bei. Damit ist das qualitative Verhalten der Vertauschungsfunktion $S_{\alpha v}\left(x-x^{\prime}\right)$ klargestellt. Eine quantitative Berechnung, die nicht von der Approximation $\varkappa(s) \sim \varkappa$ Gebrauch machen darf, wird erst im Zusammenhang mit einer Bestimmung der Eigenwerte und Matrixelemente möglich sein (Abschn. 4). Man muß dann die Summation über die Zwischenzustände $\Phi$ ausführen.

$$
\begin{gathered}
-i S_{\alpha \nu}\left(x-x^{\prime}\right)=\Sigma<\Omega\left|\psi_{\alpha}(x)\right| \Phi><\Phi\left|\psi_{\nu}^{+}\left(x^{\prime}\right)\right| \Omega> \\
+\Sigma<\Omega\left|\psi_{\nu}^{+}\left(x^{\prime}\right)\right| \Phi><\Phi\left|\psi_{\alpha}(x)\right| \Omega>, \quad \text { (3la) }
\end{gathered}
$$

wobei es für $s \neq 0$ genügt, nur über die Zustände des Hilbertraumes I zu summieren. Wenn man $S_{1}$ in der gleichen Weise berechnet, so wird allerdings auch für $s \neq 0$ ein Beitrag vom Hilbertraum II übrigbleiben; es ist daher zweckmäßig, $S_{1}$ durch Umrechnung aus $S$ zu bestimmen.

Bevor nun weitere Schlüsse aus Gl. (31) gezogen werden, soll die Frage nach der Berechtigung des für die Theorie entscheidenden Ansatzes (27) erörtert werden. Im ersten Augenblick lassen sich 
gegen diesen Ansatz in der Tat sehr schwerwiegende Einwände erheben: Wenn man den Hilbert-Raum II als einen echten Hilbert-Raum ansieht, so müßte man im Gegenteil von den ungeheuer vielen $\mathrm{Zu}$ ständen dieses Raumes einen so großen Beitrag in den Übergangselementen des Operators $\chi_{\alpha}\left(x, x^{\prime}\right)$ erwarten, daß man kaum den singulären Hauptanteil als $c$-Zahl abspalten könnte; die Vertauschungsfunktion müßte gerade in der unmittelbaren Nähe der Singularität in höchstem Maße eine ,, $q$ Zahl" sein.

Andererseits ist es eine entscheidende Grundannahme der bisherigen Quantentheorie, daß diese Abspaltung doch möglich sein solle. Denn die übliche Quantentheorie nimmt für die Vertauschungsfunktion zu gleichen Zeiten die Diracsche $\delta$-Funktion, also eine ,,c-Zahl“" an, und setzt damit ausdrücklich voraus, daß die Vertauschungsfunktion in der unmittelbaren Umgebung von $s=0$ durch eine $c$-Zahl dargestellt werden kann.

Wahrscheinlich steht diese Annahme in direktem Widerspruch zu der anderen, daß es den HilbertRaum II wirklich geben solle, und die üblichen Divergenzschwierigkeiten zeigen nur die Unvereinbarkeit der beiden Voraussetzungen. Wenn dies zutrifft, wenn man also doch gezwungen ist, einen Teil der bisherigen Voraussetzungen fallen zu lassen, so erhält man wohl noch den besten Anschluß an die bisherige Quantentheorie, wenn man die Existenz des Hilbert-Raums II im üblichen Sinne aufgibt und dafür die Annahme (27) beibehält; dies ist der physikalische Sinn des in der früheren Arbeit ${ }^{1}$ und hier unternommenen Versuchs.

Aus Gl. (31) folgt vermöge der Schwingerschen Beziehung:

$$
S_{1}(\mathfrak{r}, t)=\frac{1}{\pi} \int_{-\infty}^{+\infty} \frac{\mathrm{d} t^{\prime}}{t^{\prime}} S\left(\mathfrak{r}, t-t^{\prime}\right)
$$

die schon früher ${ }^{1}$ angegebene Formel

$$
\begin{gathered}
S_{1}\left(x-x^{\prime}\right) \approx \frac{\varkappa^{2}}{4 \pi} \gamma_{\mu} \frac{\partial}{\partial x_{\mu}} \operatorname{Im}\left[\frac{H_{1}^{(1)}(\varkappa \sqrt{s})}{\varkappa \sqrt{s}}+\frac{2 i}{\pi \varkappa^{2} s}\right. \\
\left.-\frac{i}{\pi} \lg \frac{\gamma \varkappa \sqrt{|s|}}{2}\right]-\frac{\varkappa^{3}}{4 \pi} \operatorname{Im}\left[\frac{H_{1}^{(1)}(\varkappa \sqrt{s})}{\varkappa \sqrt{s}}+\frac{2 i}{\pi \varkappa^{2} s}\right] \\
\text { für } s \lessgtr 0,
\end{gathered}
$$$$
S_{1}(0) \text { ist unbestimmt. }
$$

Die schließlich gewonnenen Ausdrücke für $S$ und $S_{1}$ haben eine gewisse Beziehung zu dem von Pauli und Villars ${ }^{3}$ vorgeschlagenen Regularisierungs-

\footnotetext{
${ }^{3}$ W. Pauli u. F. Villars, Rev. Mod. Phys. 21, 434
} [1949]. verfahren. Auch Pauli und Villars haben versucht, die Singularitäten der S-Funktion bei $s=0$ dadurch zu beseitigen, daß man die Existenz sehr schwerer Massen postuliert, deren Beiträge zu $S$ man aber dann von $S$ abzieht, statt sie zu addieren - was darauf hinausläuft, daß man hinsichtlich der Vorgänge, an denen die schweren Massen beteiligt sind, die kausale Zeitreihenfolge aufgeben muß. Daß in einer normalen Quantentheorie der Wellenfelder solche subtraktiven Glieder nicht auftreten können, wurde schon früher von verschiedenen Autoren hervorgehoben. Die hier gewonnenen Funktionen $S$ bzw. $S_{1}$ unterscheiden sich aber noch von den aus dem Pauli-Villars-Verfahren hervorgegangenen. Die Abzüge, die hier vorgenommen sind, könnten auch durch die negativen Beiträge sehr schwerer Massen allein nicht erklärt werden, was insbesondere aus dem langsamen Abfall der $S_{1^{-}}$ Funktion hervorgeht. Vielmehr müßte man sowohl im Gebiet schwerster Massen als auch bei der Masse 0 Abzüge zulassen; aber auch dann wird man (31) und (33) nicht vollständig darstellen können, wie das logarithmische Glied in (33) zeigt.

Man verlangt also mit den Gl. (31) und (33) zweifellos Beiträge vom Hilbert-Raum II, die dieser nicht leisten könnte, wenn es sich um einen echten Hilbert-Raum handelte. Wir charakterisieren den symbolischen Hilbert-Raum II daher durch die genannten negativen Beiträge, ähnlich wie etwa die Zahl $i$ durch die Eigenschaft charakterisiert wird, daß ihr Quadrat - l sein soll, owohl es eine solche Zahl natürlich nicht gibt. Irgendwelche weiteren Angaben über den symbolischen Hilbert-Raum II erweisen sich als unnötig und wohl auch als unmöglich.

Die Beiträge des symbolischen Hilbert-Raums II können in ihren physikalischen Konsequenzen ähnlich wie bei Pauli und Villars zu einem Verhalten Anlaß geben, das zunächst als eine Abweichung von der kausalen Zeitreihenfolge erscheint. Die Verhältnisse liegen aber hier günstiger als beim Regularisierungsverfahren; denn die Funktion $S$ in Gl. (31) ist ja die Grenzlösung einer klassischen Wellengleichung und insofern eine völlig kausale Fortpflanzungsfunktion. Nur die Funktion $S_{1}$ weist hier, wie überall in der Quantentheorie, andere Eigenschaften auf, die man aber - da es sich dann um Quanteneffekte handelt - besser als Folge einer endlichen Größe der Elementarteilchen denn als Abweichung von der kausalen Reihenfolge interpretieren sollte. Die Annahme der Formeln (31) und (33) 
für $S$ und $S_{1}$ bedeutet also wohl, daß alle Elementarteilchen eine endliche Ausdehnung erhalten, daß aber darüber hinaus, etwa im Bereich der näherungsweisen Gültigkeit der klassischen Wellengleichung (1), keine Abweichungen von der kausalen Zeitreihenfolge vorkommen. Durch dieses Verhalten unterscheidet sich die vorliegende Theorie auch von einem früher mit dem gleichen Ziel unternommenen Versuch des Verfassers ${ }^{4}$. Bei dem damals diskutierten Gleichungssystem mußten, wie Fier ${ }^{5}$ gezeigt hat, Abweichungen von der kausalen Zeitreihenfolge in Kauf genommen werden.

\section{Abschätzung der tiefsten Eigenwerte}

a) Spinorteilchen

Zur Bestimmung der tiefsten Eigenwerte soll das Verfahren benützt werden, das in der früheren Arbeit im Anschluß an die dort zitierten Untersuchungen von Freese und anderen ${ }^{6}$ beschrieben und auf den anharmonischen Oszillator angewendet worden ist. Man definiert zunächst die Matrixelemente von zeitlich geordneten Produkten der $\psi$-Funktionen, die zum Übergang vom Vakuum $\Omega$ zu dem gesuchten Zustand $\Phi$ gehören :

$$
\begin{aligned}
& \tau\left(x_{1} \ldots x_{m} \mid y_{1} \ldots y_{n}\right) \\
& \quad=<\Omega\left|T \psi\left(x_{1}\right) \ldots \psi\left(x_{m}\right) \psi^{+}\left(y_{1}\right) \ldots \psi^{+}\left(y_{n}\right)\right| \Phi>
\end{aligned}
$$

und ersetzt die Operator-Gleichung (1) durch ein System von Gleichungen zwischen diesen Funktionen. Dann bildet man einen zweiten Satz derartiger Funktionen (vgl. insbes. Freese ${ }^{6}$ ):

$$
\begin{aligned}
& \varphi\left(x_{1} \ldots x_{m} \mid y_{1} \ldots y_{n}\right)=\tau\left(x_{1} \ldots x_{m} \mid y_{1} \ldots y_{n}\right) \\
& \quad-\frac{1}{2} S_{F}\left(x_{1}-y_{1}\right) \tau\left(x_{2} \ldots x_{m} \mid y_{2} \ldots y_{n}\right)-\ldots(35) \\
& \quad+\frac{1}{4} S_{F}\left(x_{1}-y_{1}\right) S_{F}\left(x_{2}-y_{2}\right) \tau\left(x_{3} \ldots x_{m} \mid y_{3} \ldots y_{m}\right) \\
& \quad+\ldots-\ldots
\end{aligned}
$$

Man schließt nun das System der Differentialgleichungen zwischen den $\tau$-Funktionen durch die Annahme ab, daß alle $\varphi$-Funktionen mit einer Variabelnanzahl $m+n>N$ verschwinden sollen:

$$
\varphi\left(x_{1} \ldots x_{m} \mid y_{1} \ldots y_{n}\right)=0 \text { für } m+n>N .
$$

Die Eigenwerte des Systems sollen unter diesen Voraussetzungen um so genauer dargestellt werden, je größer die Zahl $N$ gewählt wird. Allerdings wer-

${ }^{4}$ W. Heisenberg, Z. Naturforschg. 5a, 251, 367 [1950] und 6a, 281 [1951].

5 M. Fierz, Helv. Phys. Acta 23, 731 [1950].

${ }^{6}$ E. Freese, Z. Naturforschg. 8a, 776 [1953]. Vgl. dazu auch die Arbeiten: E. Salpeter u. H. A. Bethe, Phys. Rev. 84, 1232 [1951]; M. Gell-Mann u. F. den die Rechnungen für größer werdendes $N$ bald unübersehbar kompliziert; aber vielleicht gelingt es später, geeignete Näherungsverfahren zu ersinnen.

Für die $S_{F}$-Funktionen setzen wir die aus (31) und (33) in der üblichen Weise gebildeten Funktionen ein, wobei noch speziell

$$
S(0)=S_{1}(0)=0
$$

angenommen werden soll; wenn alle Abstände $x_{i}-y_{k}$ raumartig sind, kommt es dann auf die Reihenfolge der Faktoren auf der rechten Seite von (34) nicht mehr an. Für $\varkappa$ setzt man in den niedrigsten Näherungen zweckmäßig den Energieeigenwert des tiefsten Zustandes ein. In den höheren Näherungen kann man $S_{\mathrm{F}}$ ähnlich wie in ${ }^{1}$ Gl. (37) u. f. verbessern; es ist aber die Frage, ob dadurch eine merkliche Verbesserung der Konvergenz erreicht wird.

Ferner soll der Einfachheit halber vorausgesetzt werden, daß der gesuchte Zustand $\Phi$ zum Gesamtpuls 0 und zur (gesuchten) Gesamtenergie $E$ gehört, wobei es sich in den Gleichungen formal stets um die Differenzenergie bzw. Differenzimpuls zwischen $\Phi$ und dem Vakuum handelt. Wir definieren aber Energie und Impuls des Vakuums als Null.

Die $\tau$ - bzw. $\varphi$-Funktionen hängen dann vom mittleren Ort $\mathfrak{r}=\frac{\mathrm{r}_{1}+\mathrm{r}_{2} \ldots}{n+m}$ überhaupt nicht, und von der mittleren Zeit $t=\frac{t_{1}+t_{2}+\ldots}{n+m}$ in der Form $e^{i E t} \mathrm{ab}$.

Wenn die Spinorindizes ausdrücklich angeschrieben werden müssen, so sollen sie dem Zeichen $\tau$ bzw. $\varphi$ in der Reihenfolge der zugehörigen Ortskoordinaten angefügt werden, d. h. z. B. der dritte Spinorindex gehört zu $x_{3}$, bzw., wenn die Ortskoordinaten lauten: $x_{1}, x_{2} / y_{1}$ zu $y_{1}$; über paarweise auftretende Indizes wird stets summiert.

Unter diesen Voraussetzungen behandeln wir zunächst die Näherung $N=1$. Aus Gl. (1) folgt:

$$
\gamma_{\mu}^{\alpha \beta} \frac{\partial \tau_{\beta}(x)}{\partial x_{\mu}}=-l^{2} \tau_{\alpha \gamma \gamma}(x x \mid x) .
$$

Ferner aus (35) und (36)

$$
\begin{array}{r}
\varphi_{\alpha \gamma \gamma}(x x \mid x)=\tau_{\alpha \gamma \gamma}(x x \mid x)-\frac{1}{2} S_{F}^{\alpha \gamma}(0) \tau_{\gamma}(x) \\
-\frac{1}{2} S_{F}^{\gamma \gamma}(0) \tau_{\alpha}(x)=0,
\end{array}
$$

Low, Phys. Rev. 84, 350 [1951]; M. Gell-Mann u. M. L. Goldberger, Phys. Rev. 87, 218 [1952]; J. Schwinger, Proc. Nat. Acad. Sci., Wash. 37, 452, 455 [1951]; W. Zimmermann, Z. Phys. 135, 473 [1953] und Nuovo Cim. im Erscheinen; K. Symanzik, Dissert. Göttingen 1954, im Erscheinen. 
d. h. aber nach (37)

$$
\begin{aligned}
\tau_{\alpha \gamma \gamma}(x x \mid x)=0 \quad \text { und } \\
\gamma_{\mu} \frac{\partial \tau(x)}{\partial x_{\mu}}=0 ; \quad \frac{\partial \tau}{\partial x_{4}}=0 ; \quad E=0 .
\end{aligned}
$$

In dieser Näherung gibt es also nur Elementarteilchen der Ruhmasse 0. Das ist verständlich, da hier die Wechselwirkung noch ausgeschaltet ist, die allein eine Ruhmasse hervorbringen kann.

Die nächste Näherung für die Masse des leichtesten Spinorteilchens erhält man aus der Annahme
$N=3$. Dann bleibt die Gl. (38) ungeändert richtig, $\tau_{\alpha \gamma \gamma}(x x \mid x)$ kann aber nicht mehr aus Gl. (39) bestimmt werden. Vielmehr wird man zur Bestimmung von $\tau_{\alpha \gamma \gamma}(x x \mid x)$ etwa die aus (1) folgende Gleichung:

$\gamma_{\mu}^{\alpha \beta} \frac{\partial}{\partial x_{\mu}} \tau_{\beta \gamma \gamma}\left(x x^{\prime} \mid x^{\prime}\right)=-l^{2} \tau_{\alpha \delta \gamma \delta \gamma}\left(x x x^{\prime} \mid x x^{\prime}\right)$

heranziehen, indem man nun (unter Berücksichtigung von (37)):

$\varphi_{\alpha \delta \gamma \delta \gamma}\left(x x x^{\prime} \mid x x^{\prime}\right)=\tau_{\alpha \delta \gamma \delta \gamma}\left(x x x^{\prime} \mid x x^{\prime}\right)-\frac{1}{2} S_{F}^{\gamma \delta}\left(x^{\prime}-x\right) \tau_{\alpha \delta \gamma}\left(x x \mid x^{\prime}\right)-\frac{1}{2} S_{F}^{\gamma \delta}\left(x-x^{\prime}\right) \tau_{\alpha \gamma \delta}\left(x x^{\prime} \mid x\right)$
$-\frac{1}{2} S_{F}^{\alpha \gamma}\left(x-x^{\prime}\right) \tau_{\delta \gamma \delta}\left(x x^{\prime} \mid x\right)+\frac{1}{4} S_{F}^{\gamma \delta}\left(x^{\prime}-x\right) S_{F}^{\delta \gamma}\left(x-x^{\prime}\right) \tau_{\alpha}(x)+\frac{1}{4} S_{F}^{\gamma \delta}\left(x^{\prime}-x\right) S_{F}^{\alpha \gamma}\left(x-x^{\prime}\right) \tau_{\delta}(x)=0$

annimmt. Setzt man $\tau_{\alpha \delta \gamma \delta \gamma}\left(x x x^{\prime} \mid x x^{\prime}\right)$ aus (43) in Gl. (42) ein, so erscheinen auf der rechten Seite Glieder mit $\tau\left(x x^{\prime} \mid x\right)$ und solche mit $\tau(x)$. Nur die letzteren sind der mathematische Ausdruck dafür, daß in der Umgebung eines Spinorteilchens immer noch durch die Wechselwirkung ein virtuelles Paar solcher Teilchen erzeugt und dadurch eine Ruhmasse hervorgebracht wird. Wir wollen, um die Rechnung nicht unnötig zu komplizieren, nur diese Glieder auf der rechten Seite von (42) beibehalten und die Glieder mit $\tau\left(x x^{\prime} \mid x\right)$ vernachlässigen, weil der dadurch verursachte Fehler im Eigenwert wohl die auch sonst vorhandene Ungenauigkeit des Eigenwertes in der Näherung $N=3$ nicht übersteigt. In dieser Näherung entsteht also aus (42) die Beziehung:

$\gamma_{\mu}^{\alpha \beta} \frac{\partial}{\partial x_{\mu}} \tau_{\beta \gamma \gamma}\left(x x^{\prime} \mid x^{\prime}\right)=\frac{l^{2}}{4} S_{F}^{\gamma \delta}\left(x^{\prime}-x\right) S_{F}^{\delta \gamma}\left(x-x^{\prime}\right) \tau_{\alpha}(x)$

$$
+\frac{l^{2}}{4} S_{F}^{\gamma \delta}\left(x^{\prime}-x\right) S_{F}^{\alpha \gamma}\left(x-x^{\prime}\right) \tau_{\delta}(x) .
$$

Führt man nun die Differenz $x^{\prime}-x$ als neue Variable ein:

so kann man

$$
y=x^{\prime}-x,
$$

$$
\tau_{\delta}(x)=e^{i E t} \tau_{\delta} \text { und } \tau_{\alpha \gamma \gamma}\left(x x^{\prime} / x^{\prime}\right)=e^{i E t} \sigma_{\alpha}(y)
$$

setzen und erhält aus (44):

$E \gamma_{4}^{\alpha \beta} \sigma_{\beta}(y)-\gamma_{\mu}^{\alpha \beta} \frac{\partial}{\partial y_{\mu}} \sigma_{\beta}(y)$

$=\frac{l^{2}}{4} S_{F}^{\gamma \delta}(y) S_{F}^{\delta \gamma}(-y) \tau_{\alpha}+\frac{l^{2}}{4} S_{F}^{\alpha \gamma}(-y) S_{F}^{\gamma \delta}(y) \tau_{\delta}$.

Beschränkt man sich zunächst auf den Teil des Raumes, in dem der Vektor $y$ raumartig ist - und das wird für die folgenden Rechnungen genügen so kann man $S_{F}$ durch $S_{1}$ ersetzen und unmittelbar aus Gl. (33) entnehmen. Dabei erhält man aus (33) durch eine einfache Umformung:

$S_{1}(x)=-\frac{\varkappa^{4}}{2 \pi} \gamma_{\mu} x_{\mu} f(\varkappa \sqrt{-s})+\frac{\varkappa^{3}}{4 \pi} g(\varkappa \sqrt{-s})$,

wobei

$f(z)=-\frac{H_{1}^{(1)}(i z)}{z^{3}}+\frac{i H_{0}^{(1)}(i z)}{2 z^{2}}-\frac{2}{\pi z^{4}}+\frac{1}{2 \pi z^{2}}$

und $\quad g(z)=\frac{H_{1}^{(1)}(i z)}{z}+\frac{2}{\pi z^{2}}$.

Aus (47) wird dann:

$$
\begin{aligned}
& E \gamma_{4}^{\alpha \beta} \sigma_{\beta}(y)-\gamma_{\mu}^{\alpha \beta} \frac{\partial}{\partial y_{\mu}} \sigma_{\beta}(y) \\
& \quad=\frac{5 l^{2} \varkappa^{6}}{64 \pi^{2}}\left(g^{2}\left(\varkappa \sqrt{y_{\mu}^{2}}\right)-4 \varkappa^{2} y_{\mu}^{2} f^{2}\left(\varkappa \sqrt{y_{\mu}^{2}}\right)\right) \tau_{\alpha} .
\end{aligned}
$$

Für die weitere Behandlung ist es zweckmäßig, vom Ortsraum zum Impulsraum überzugehen und eine Fourier-Darstellung für die rechte Seite von (51) zu suchen. $\mathrm{Zu}$ diesem Zweck wurde die rechte Seite als Funktion von $\varkappa \sqrt{y_{\mu}^{2}}$ numerisch ausgewertet; es stellte sich dabei heraus, daß das Resultat durch die Differenz zweier Funktionen vom Typus $g(z)$ genügend genau approximiert werden konnte. Gl. (51) geht damit über in:

$$
\begin{aligned}
E \gamma_{4}^{\alpha \beta} \sigma_{\beta}(y) & -\gamma_{\mu}^{a \beta} \frac{\partial}{\partial y_{\mu}} \sigma_{\beta}(y) \\
& \approx \frac{l^{2} \varkappa^{6}}{64 \pi^{2}}\left[9 g\left(3 \varkappa \sqrt{y_{\mu}^{2}}\right)-g\left(\varkappa \sqrt{y_{\mu}^{2}}\right)\right] \tau_{\alpha} .
\end{aligned}
$$

Für die rechte Seite erhält man nunmehr die Fourier-Darstellung

$-\frac{l^{2} \varkappa^{4}}{256 \pi^{5}} \oint e^{i k_{\mu} y_{\mu}} \mathrm{d} k\left[\frac{1}{k_{\mu}^{2}+9 \varkappa^{2}}-\frac{1}{k_{\mu}^{2}+\varkappa^{2}}\right] \tau_{\alpha}$, 
wobei in der komplexen $k_{4}$-Ebene um die Pole auf der $+i$-Achse im positiven, um die der $-i$-Achse im negativen Sinne integriert werden muß.

Schließlich ergibt sich für $\sigma_{\beta}(y)$ :

$$
\begin{aligned}
& \sigma_{\beta}(y)=-\frac{l^{2} \varkappa^{4}}{256 \pi^{5}} \oint e^{i k_{\mu} y_{\mu}} \mathrm{d} k \\
& . \frac{\left(E-i k_{4}\right) \gamma_{4}^{\beta \alpha}-i k_{l} \gamma_{l}^{\beta \alpha}}{\left(E-i k_{4}\right)^{2}-k_{l}{ }^{2}}\left[\frac{1}{k_{\mu}^{2}+9 \varkappa^{2}}-\frac{1}{k_{\mu}^{2}+\varkappa^{2}}\right] \tau_{\alpha} .
\end{aligned}
$$

Der Integrationsweg in der komplexen $k_{4}$-Ebene ist aus Abb. 2 ersichtlich; er ist so gewählt, daß Gl. (52) befriedigt wird, $\sigma_{\beta}(0)$ endlich bleibt und $\sigma_{\beta}(y)$ im Unendlichen $y_{\mu}^{2} \rightarrow \infty$ verschwindet.

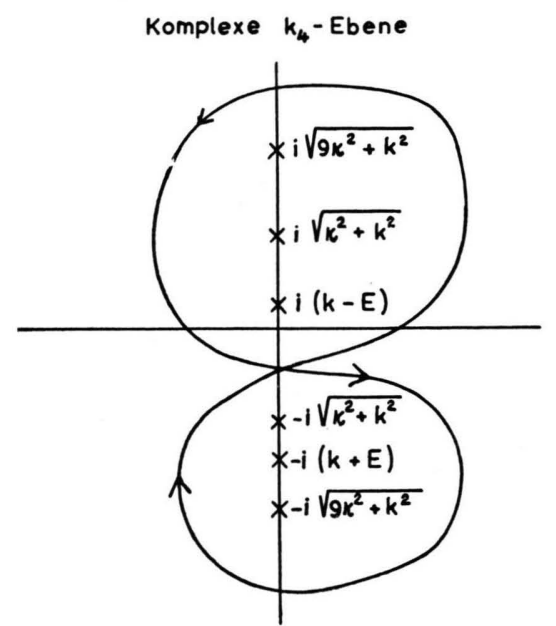

Abb. 2. Integrationsweg in der komplexen $k_{4}$-Ebene.

Man erkennt aus (54), daß die Abhängigkeit von $y_{\mu}$ in $\sigma_{\beta}(y)$ komplizierter ist als bei den Vertauschungsfunktionen $S$; denn hier ist jetzt das Koordinatensystem ausgezeichnet, in dem das Teilchen, das durch den tiefsten Zustand dargestellt wird, ruht. Für die weiteren Rechnungen braucht man wegen (38) und (46) nur noch den Wert von $\sigma_{\beta}(y)$ an der Stelle $y_{\mu}=0$.

Für $\sigma_{\beta}(0)$ ergibt sich aus (54)

$$
\begin{aligned}
\sigma_{\beta}(0)=-\frac{l^{2} \varkappa^{4} E \gamma_{4}{ }^{\beta \alpha} \tau_{\alpha}}{32 \pi^{3}} \int_{0}^{\infty} k^{2} \mathrm{~d} k \\
\cdot\left[\frac{E^{2}-9 \varkappa^{2}-2 k^{2}+2 k \sqrt{k^{2}+9 \varkappa^{2}}}{\left[\left(E^{2}-9 \varkappa^{2}\right)^{2}-4 E^{2} k^{2}\right\rfloor \sqrt{k^{2}+9 \varkappa^{2}}}\right. \\
\left.-\frac{E^{2}-\varkappa^{2}-2 k^{2}+2 k \sqrt{k^{2}+\varkappa^{2}}}{\left[\left(E^{2}-\varkappa^{2}\right)^{2}-4 E^{2} k^{2}\right] \sqrt{k^{2}+\varkappa^{2}}}\right] .
\end{aligned}
$$

Für die Masse $\varkappa$, die zum erstenmal in Gl. (28) aufgetreten ist, wird man zweckmäßig - ähnlich wie in den Rechnungen zum anharmonischen Oszillator in der früheren Arbeit ${ }^{1}$ - den tiefsten Eigenwert einsetzen, also

$$
\varkappa=E
$$

fordern. Dann vereinfacht sich das Integral der rechten Seite von (55) zu

$$
\begin{gathered}
Z=\int_{0}^{\infty} \zeta^{2} \mathrm{~d} \zeta\left[\frac{-8-2 \zeta^{2}+2 \zeta \sqrt{9+\zeta^{2}}}{\left(64-4 \zeta^{2}\right) \sqrt{9+\zeta^{2}}}\right. \\
\left.+\frac{-2 \zeta^{2}+2 \zeta \sqrt{1+\zeta^{2}}}{4 \zeta^{2} \sqrt{1+\zeta^{2}}}\right]
\end{gathered}
$$

$=1+24 \lg 2-\frac{63}{4} \lg 3=0,332$.

Aus den Gl. (38) und (55) folgt jetzt sofort

$$
\begin{aligned}
E \gamma_{4} \tau & =\frac{E l^{4} \varkappa^{4}}{32 \pi^{3}} Z \gamma_{3} \tau \text { und } \\
\varkappa^{4} l^{4} & =32 \pi^{3} / Z ; \quad E=\varkappa=7,45 / l .
\end{aligned}
$$

Die Theorie ergibt also in dieser Näherung $(N=3)$ für die Masse des leichtesten Spinorteilchens etwa das 7,5-fache der reziproken charakteristischen Länge $l$. Setzt man für $l$ die Compton-Wellenlänge des $\pi$-Mesons ein, so erhält man daher etwa die Masse des Protons. Natürlich bedeutet eine solche numerische Koinzidenz wenig, da die Ausgangsgleichung (1) sicher noch nicht die richtige Form haben wird.

\section{b) Bose-Teilchen}

Ein Zustand, dessen Hilbert-Vektor aus dem des Vakuums durch Anwendung einer geraden Anzahl von $\psi$-Operatoren hervorgeht, stellt dann ein BoseTeilchen dar, wenn es sich um einen diskreten stationären Zustand handelt. Man wird die tiefsten stationären Zustände dieser Art erhalten, wenn man die Methode des letzten Abschnitts auf $\tau$-Funktionen mit einer geraden Anzahl von Variabeln anwendet.

In der Näherung $N=2$ wird man allerdings nur Zustände erhalten können, in denen zwei Spinorteilchen der Ruhmasse 0 vorhanden sind; eine Bindung ist hier noch nicht möglich, da die Energie des Zustandes ja sicher nicht kleiner als Null sein kann. Erst in der Näherung $N=4$ kann sich die Wechselwirkung bemerkbar machen. In dieser Näherung kann man von den beiden folgenden aus (1) gewonnenen Gleichungen ausgehen:

$$
\begin{aligned}
& \gamma_{\mu}^{\alpha \beta} \frac{\partial}{\partial x_{\mu}} \tau_{\beta \gamma}(x y)=-l^{2} \tau_{\alpha \delta \gamma \delta}(x x \mid y x), \\
& \gamma_{\mu}^{\varepsilon \eta} \frac{\partial}{\partial y_{\mu}} \tau_{\alpha \delta \varepsilon \delta}(x x \mid y x)=-l^{2} \tau_{\alpha \delta \gamma \eta \delta \gamma}(x x x y \mid y x y) .
\end{aligned}
$$


Aus $\varphi(x x y \mid y x y)=0$ gewinnt man ähnlich wie früher, unter Vernachlässigung der Terme mit $\tau(x x \mid y x)$ in dieser Gleichung, die Beziehung:

$$
\begin{array}{r}
4 \tau_{\alpha \delta \gamma \eta \delta \gamma}(x x y \mid y x y) \approx-S_{F}^{\gamma \delta}(y-x) S_{F}^{\alpha \eta}(x-y) \tau_{\delta \gamma}(x y) \\
-S_{F}^{\gamma \delta}(y-x) S_{F}^{\alpha \gamma}(x-y) \tau_{\delta \eta}(x y)-\ldots(60)
\end{array}
$$

Schreibt man nun, mit

$$
z=y-x, \quad x_{4}=i t
$$

für die $\tau$-Funktionen abkürzend

$\tau_{\alpha \beta}(x y)=e^{i E t} \tau_{\alpha \beta}(z) ; \quad \tau_{\alpha \delta \beta \delta}(x x \mid y x)=e^{i E t} \sigma_{\alpha \beta}(z)$,

so erhält man schließlich als Eigenwertgleichung zur Bestimmung von $E$ aus (59) und (60):

$$
\begin{aligned}
E \gamma_{4}^{\alpha \beta} \tau_{\beta \gamma}(z)-\gamma_{\mu}^{\alpha \beta} & \frac{\partial}{\partial z_{\mu}} \tau_{\beta \gamma}(z)=-l^{2} \sigma_{\alpha \gamma}(z), \\
4 \gamma_{\mu}^{\delta \eta} \frac{\partial}{\partial z_{\mu}} \sigma_{\alpha \delta}(z) & =l^{2} S_{F}^{\alpha \eta}(-z) S_{F}^{\varepsilon \delta}(z) \tau_{\delta \varepsilon}(z) \\
& +l^{2} S_{F}^{\alpha \varepsilon}(-z) S_{F}^{\varepsilon \delta}(z) \tau_{\delta \eta}(z), \\
& +l^{2} S_{F}^{\alpha \eta}(-z) S_{F}^{\delta \varepsilon}(-z) \tau_{\varepsilon \delta}(-z) \\
& +l^{2} S_{F}^{\alpha \varepsilon}(-z) S_{F}^{\delta \eta}(-z) \tau_{\varepsilon \delta}(-z) \\
& +l^{2} S_{F}^{\delta \varepsilon}(-z) S_{F}^{\varepsilon \delta}(z) \tau_{\alpha \eta}(z) \\
& +l^{2} S_{F}^{\delta \eta}(-z) S_{F}^{\varepsilon \delta}(z) \tau_{\alpha \varepsilon}(z) .
\end{aligned}
$$

Für die Funktionen $S_{F}^{\alpha \beta}$ muß man im Raum $z_{\mu}^{2}>0$ wieder die Werte aus (48) mit $\varkappa=7,45 / l$ nach $(58)$ einsetzen. Man erkennt, daß der Ausdruck

$$
g^{2}\left(\varkappa \sqrt{z_{\mu}^{2}}\right)-4 \varkappa^{2} z_{\mu}^{2} f^{2}\left(\varkappa \sqrt{z_{\mu}^{2}}\right),
$$

der schon bei der Bestimmung der Masse des Spinorteilchens eine entscheidende Rolle gespielt hat, und andere ähnlich gebaute Ausdrücke hier als eine Art potentieller Energie zwischen den beiden virtuellen Spinorteilchen eingehen, die sie eventuell zu einem Bose-Teilchen binden.

Die numerische Auflösung der Eigenwertgleichung (62), die etwa nach dem von Salpeter ${ }^{7}$ entwickelten Verfahren erfolgen könnte, soll hier nicht versucht werden, da Gl. (62) wegen der zwei Spinorindizes und der Auszeichnung der Zeitachse reichlich kompliziert ist und da die Eigenschaften der speziellen Gleichung (1) wohl auch nicht von besonderem Interesse sind. Obwohl also noch nicht entschieden werden kann, ob die Gl. (62) zu einem diskreten Eigenwert und damit zu einem Bose-Teilchen führt, ob es sich dabei um ein Teilchen vom Spin 0 oder 1 handelt, genügt (62) doch, um zu

${ }^{7}$ E. E. Salpeter, Phys. Rev. 84, 1226 [1951]. zeigen, daß eine Wellengleichung vom Typus (1) grundsätzlich sowohl Spinorteilchen als auch BoseTeilchen liefern kann.

\section{Erhaltungssätze}

Die Unterscheidung zweier Gruppen von Elementarteilchen, die als Bosonen und Fermionen bezeichnet werden können, deutet bereits auf die Existenz einer Anzahl von Erhaltungssätzen und Auswahlregeln hin, die noch kurz besprochen werden sollen.

Aus der Lorentz-Invarianz der Gl. (1) folgen zunächst die bekannten zehn Erhaltungssätze für Energie, Impuls, Drehimpuls und Schwerpunktbewegung.

Außerdem besteht Invarianz gegenüber der Transformation

$$
\psi \rightarrow \psi e^{i a},
$$

woraus die Erhaltung der „,Ladung“ folgt (die so definierte Ladung braucht natürlich nichts mit der speziellen elektrischen oder nukleonischen Ladung der bekannten Elementarteilchen zu tun zu haben). Man kann also jeden diskreten stationären Zustand, d. h. jedes Elementarteilchen, durch seine Ladung charakterisieren. Der Hilbert-Vektor eines Zustandes der Ladung $n$ geht aus dem des Vakuums durch einen Operator hervor, der additiv aus Produkten von $\psi$ - und $\psi^{+}$-Operatoren zusammengesetzt ist, wobei die Anzahl der $\psi^{+}$-Operatoren in jedem dieser Produkte genau um $n$ größer ist als die der $\psi$-Operatoren. Das in 4a) untersuchte Fermi-Teilchen hat also die Ladung 1 und den Spin $\left(\frac{1}{2}\right)$, das in 4 b) untersuchte Bose-Teilchen die Ladung 0. Alle Teilchen mit ungerader Ladung genügen der FermiStatistik und besitzen halbzahligen Spin, alle Teilchen mit gerader Ladung genügen der Bose-Statistik und besitzen ganzzahligen Spin.

Gl. (1) ist ferner invariant gegenüber der Ladungsumkehr; man erhält aus jedem Teilchen das zugehörige ,Antiteilchen“, indem man im HilbertVektor zu dem Operator, der auf den Vakuumvektor angewendet wird, den hermitesch konjugierten bildet und diesen auf den Vakuumvektor anwendet.

Schließlich ist Gl. (1) noch invariant gegenüber der Operation

$$
x_{\lambda}, \gamma_{\lambda} \rightarrow-x_{\lambda},-\gamma_{\lambda},
$$

was zu den bekannten Paritätseigenschaften führt, auf die aber hier nicht näher eingegangen werden soll. 
Im ganzen ist aber das System von Elementarteilchen, das aus Gl. (1) entspringt, offenbar einfacher und damit weniger reichhaltig als das der wirklichen Elementarteilchen; denn bei den wirklichen Elementarteilchen gibt es mindestens zwei Erhaltungssätze der Ladung (Elektrische Ladung und Nukleonenladung), außerdem hängt die Geradzahligkeit der Ladung nicht unmittelbar mit der Frage nach Halb- oder Ganzzahligkeit des Spins zusammen. Die Wellengleichung der wirklichen Materie wird also wohl etwas komplizierter sein müssen als Gl. (1).

Dabei ist hervorzuheben, daß es nicht etwa anginge, eine Gruppe von Elementarteilchen aus einer Gleichung vom Typus (1), eine andere Gruppe aus einer anderen derartigen Gleichung herleiten zu wollen. Denn das wäre nur möglich, wenn es zwischen den beiden Gruppen keinerlei Wechselwirkung gäbe. In Wirklichkeit gibt es aber Wechselwirkung zwischen allen bekannten Elementarteilchen, es muß also einen einheitlichen Hilbert-Raum geben, der alle Elementarteilchen als diskrete stationäre Zustände enthält. Wahrscheinlich kommt in der wirklichen Wellengleichung der Materie neben den Variabeln $x$ und $\alpha$ (Spinindex) noch mindestens eine weitere Variable, z. B. ein Index für den Isotopenspin, vor.

Eine besonders charakteristische Eigenschaft des Systems der wirklichen Elementarteilchen besteht darin, daß es einige Elementarteilchen verschwindender oder nahezu verschwindender Ruhmasse (Lichtquanten, Neutrinos, Elektronen) enthält. Die Ausgangsgleichung wird wohl recht spezielle Eigenschaften aufweisen müssen, um solche Lösungen zuzulassen. Die Existenz von Bosonen der Ruhmasse Null ist dabei die Voraussetzung für Kräfte großer Reichweite, also für die ganze Elektrodynamik. Die Notwendigkeit, auch solche Teilchen aus der Ausgangsgleichung herzuleiten, schränkt die Möglichkeiten für die Wellengleichung der Materie entscheidend ein.

\section{Kritik der Methode}

Die in Abschnitt 4 angewendete Methode setzt voraus, daß die zu berechnenden Eigenwerte mit wachsendem $N$ gegen endliche Grenzwerte konvergieren. Es soll nun zunächst plausibel gemacht werden, daß die in (35) definierten $\varphi$-Funktionen unter gewissen Voraussetzungen tatsächlich mit wachsen$\operatorname{dem} N$ beliebig klein werden. $Z u$ diesem $Z$ weck betrachten wir die $\tau$ - bzw. $\varphi$-Funktionen auf einem bestimmten Schnitt $t=$ const, so daß sie dort nur von den räumlichen Koordinaten der Teilchen abhängen; von diesen räumlichen Koordinaten gehen wir durch Fourier-Transformation zu den Impulsen über, die $\tau$ - bzw. $\varphi$-Funktionen hängen also jetzt von den Impulsen der Teilchen und von der gemeinsamen Zeit ab. Wenn der gesuchte Zustand $\Phi$ keine allzugroße Energie hat und wenn man sich seinen Hilbert-Vektor dargestellt denkt durch die Anwendung von Summen von Operatorprodukten der Art $\psi_{\alpha}(\mathfrak{p}) \psi_{\beta}\left(\mathfrak{p}^{\prime}\right) \psi_{\gamma}{ }^{+}\left(\mathfrak{p}^{\prime \prime}\right)$ auf den Vakuumvektor, so werden, wie später noch näher begründet werden wird, unter diesen Operatoren die $\psi(\mathfrak{p})$, $\psi^{+}(\mathfrak{p})$ mit sehr großen Impulsen nur mit sehr kleiner Intensität vorkommen. Wir denken uns nun weiter die erwähnte Fourier-Zerlegung in einem endlichen Volumen $V$ durchgeführt, so daß die Impulse $\mathfrak{p}$ nur diskrete Werte annehmen können. Betrachtet man jetzt $\tau$-Funktionen mit einer hohen Zahl $N$ von Faktoren, so kann wegen des Pauli-Prinzips jeder Faktor $\psi_{\alpha}(\mathfrak{p})$ zu einem bestimmten Wertepaar $\mathfrak{p}, \alpha$ nur einmal vorkommen (zu einem bestimmten Wert von $\mathfrak{p}$ also wegen der vier möglichen Werte von $\alpha$ viermal), da sonst die $\tau$ - und die $\varphi$-Funktion verschwindet. Steigert man die Zahl $N$ der Faktoren, so muß man also zu immer höheren Werten von $\mathfrak{p}$ übergehen, schließlich zu $\mathfrak{p}$-Werten, deren zugehörige $\psi(\mathfrak{p})$-Operatoren in der Darstellung von $\Phi$ praktisch nicht mehr vorkommen. Dann ergibt ein derartiger Faktor der Form $T \varphi_{\alpha}(\mathfrak{p}) \psi_{\beta}{ }^{+}(\mathfrak{p})$ in der $\tau$ Funktion einfach den Vakuumerwartungswert dieses Faktors, und die zugehörige $\varphi$-Funktion verschwindet, da ja in (35) eben die Vakuumerwartungswerte derartiger Faktoren abgezogen werden. Wenn also die Operatoren $\psi(\mathfrak{p})$ mit sehr großen Impulsen im Hilbert-Vektor $\Phi$ nur mit sehr kleinen Amplituden vorkommen, so werden auch die $\varphi$ Funktionen mit sehr großen $N$ schließlich praktisch verschwinden, und man wird umgekehrt den richtigen Eigenwert sehr gut annähern, wenn man für hinreichend große $N \varphi=0$ setzt. Andererseits dürfte gerade die Tatsache, daß die $S_{1}$-Funktionen in der Umgebung von $s=0$ nur noch eine logarithmische Singularität aufweisen, dafür sorgen, daß die $\psi(\mathfrak{p})$ mit sehr großen $\mathfrak{p}$-Werten tatsächlich nur noch mit ganz kleiner Amplitude auftreten.

Bei dieser Gelegenheit sei noch auf eine Besonderheit des in Abschnitt 4 verwandten Näherungsverfahrens hingewiesen. Bei strenger Rechnung sollten die $\tau$-Funktionen in der Nähe der kritischen Lichtkegel noch die logarithmische Singularität der 
$S_{F}$-Funktionen aufweisen, die $\varphi$-Funktionen sollten dort glatter sein. In jedem einzelnen Schritt des Näherungsverfahrens sieht dies aber anders aus: Zwar ist $\varphi_{N+2}=0$, und $\tau_{N+2}$ verhält sich, wie eben beschrieben; aber die niedrigeren $\tau$-Funktionen $\tau_{N}$, $\tau_{N-2}$ usw. weisen keine logarithmische Singularität mehr auf, während die $\varphi_{N}, \varphi_{N-2}$ usw. sie besitzen. Dieses Paradoxon klärt sich durch die Feststellung auf, daß es bei einem niedrigen Wert von $N$ ohne Verlust an Genauigkeit zulässig wäre, die logarithmische Spitze von $S_{F}$ etwas abzurunden (um so weniger, je höher $N$ ist), so daß dann weder die $\tau$ - noch die $\varphi$-Funktionen die logarithmische Singularität zeigen, die erst mit wachsenden $N$-Werten allmählich in Erscheinung träte.

Die in diesem Abschnitt angestellten Überlegungen geben übrigens nachträglich die Berechtigung dafür, daß bei der Ableitung der Vertauschungsfunktion in Gl. (28) die variable Masse $\varkappa(s)$ einfach durch eine konstante Masse $*$ ersetzt wurde. Es kommt für die Konvergenz des Verfahrens ja nur darauf an, daß für hinreichend hohe Impulse $\mathfrak{p}$ die in (35) auftretende Funktion $S_{F}(\mathfrak{p})$ durch den Vakuumerwartungswert von $T \psi_{\alpha}(\mathfrak{p}) \psi_{\beta}^{+}(\mathfrak{p})$ gegeben ist, bzw. daß das Verhalten von $S_{F}(x)$ in der Nähe von $x=0$ mit dem entsprechenden Vakuumerwar- tungswert übereinstimmt. Das Verhalten von $S_{F}$ bei kleinen $\mathfrak{p}$ - bzw. großen $x$-Werten kann nur für die Schnelligkeit der Konvergenz des Verfahrens von Bedeutung sein, nicht aber für die Konvergenz selbst.

Schließlich sei noch einmal darauf hingewiesen, da $\beta$ es in einer Theorie vom Typus (1) keine Elementarteilchen ohne Wechselwirkung gibt, daß die Gl. (1) vielmehr nicht nur die Massen, sondern auch die Wechselwirkung der Elementarteilchen vollständig festlegt. Eine Berechnung der Wechselwirkung soll hier nicht mehr vorgenommen werden. Qualitativ kann man aber auch ohne Rechnung vermuten, daß z. B. der größte Beitrag zu den Kräften zwischen den Spinorteilchen der Masse $7,45 / l$ von den leichtesten Bose-Teilchen herrühren wird. Die Reichweite der Kräfte zwischen den Spinorteilchen dürfte also unmittelbar durch den tiefsten diskreten Eigenwert von Gl. (62) bestimmt sein. Andererseits werden die Kräfte zwischen den Bose-Teilchen jedenfalls zum Teil durch die Spinorteilchen geliefert, und diese Kräfte haben eine Reichweite von der Größenordnung $l / 7,5$. Auch die Zahlkonstanten, die den Werten $g^{2} / \hbar c$ der Mesonentheorie bzw. $e^{2} / \hbar c$ der Elektronentheorie entsprechen, sollten sich aus Gl. (1) im Prinzip streng berechnen lassen.

\title{
Eine massenspektrographische Neubestimmung der Massen von ${ }^{\mathrm{I}} \mathrm{H},{ }^{2} \mathrm{D},{ }^{4} \mathrm{He},{ }^{\mathrm{I}} \mathrm{C}$ und ${ }^{\mathrm{r}} \mathrm{N}$
}

\author{
Von J. Mattauch und R. Bieri* \\ Aus dem Max-Planck-Institut für Chemie, Mainz \\ Otto Hahn zum 75. Geburtstag gewidmet \\ (Z. Naturforschg. 9a, 303-323 [1954]; eingegangen am 12. Februar 1954)
}

\begin{abstract}
Nach der massenspektrographischen Dublettmethode werden die Massen von ${ }^{1} \mathrm{H},{ }^{2} \mathrm{D}$, ${ }^{4} \mathrm{He},{ }^{12} \mathrm{C}$ und ${ }^{14} \mathrm{~N}$ mit einer Genauigkeit von 0,3 bis $0,5 \mathrm{ppm}$ (Teilen in einer Million) ermittelt. Bei der Herstellung und Ausmessung der Aufnahmen wurden die Versuchsbedingungen weitgehend variiert, so da $B$ man hoffen konnte, möglichst viele Ursachen systematischer Fehler zu erfassen. Die Auswertung erfolgte soweit als möglich voraussetzungslos nach einer früher beschriebenen Methode mit Hilfe von Dispersionslinien bekannter Masse auf der gleichen Aufnahme. Dabei wurde eine Abhängigkeit des Dispersionskoeffizienten von der magnetischen Feldstärke festgestellt, die bei älteren Dublettmessungen systematische Fehler verursacht hatte. Aus den sehr gut miteinander verträglichen Messungen einer Gruppe von 7 voneinander unabhängigen Dubletts ergaben sich nach dem Gaußschen Ausgleichsverfahren für die 4 durch sie überbestimmten Massenüberschüsse folgende Werte, ausgedrückt in $(\mathrm{mME} \pm \mu \mathrm{ME}):\left({ }^{1} \mathrm{H}-1\right)=(8,145.9$ $\pm 0,5),\left({ }^{2} \mathrm{D}-2\right)=(14,744.4 \pm 0,9),\left({ }^{4} \mathrm{He}-4\right)=(3,879.7 \pm 1,6), \quad\left({ }^{12} \mathrm{C}-12\right)=(3,823.1 \pm 3,3)$. Durch ein weiteres Dublett wurde der Massenüberschuß $\left({ }^{14} \mathrm{~N}-14\right)=(7,515.0 \pm 4,9)$ gefunden. Messungen, die auch diesen Wert kontrollieren sollten, zeigten eine Verfälschung durch eine bisher unaufgeklärte Fehlerursache. Aus den hier gefundenen Massenüberschüssen und unter Hinzunahme der Bindungsenergie des Deuterons werden die Masse des Neutrons, die Bindungsenergien der Kerne ${ }^{4} \mathrm{He},{ }^{12} \mathrm{C},{ }^{14} \mathrm{~N},{ }^{16} \mathrm{O}$ sowie andere Daten von kernphysikalischem Interesse berechnet.
\end{abstract}

* Die vorliegende Arbeit enthält wesentliche Bestandteile der Dissertation von R. Bieri (D 77). 